\title{
RCP induces FAK phosphorylation and ovarian cancer cell invasion with inhibition by curcumin
}

\author{
So Ra Choe ${ }^{1}$, Yu Na Kim ${ }^{1}$, Chang Gyo Park' ${ }^{1}$ Kyung Hwa Cho ${ }^{1}$, Do Yeun Cho ${ }^{2}$ and Hoi Young Lee ${ }^{1}$
}

\begin{abstract}
Rab coupling protein (RCP) aggravates cancer cell metastasis and has been implicated in various cancer patient outcomes. Recently, we showed that RCP induces Slug expression and cancer cell invasion by stabilizing the $\beta 1$ integrin protein. In the present study, we demonstrated that FAK is implicated in RCP-induced EGFR phosphorylation and ovarian cancer cell invasion with inhibition by curcumin. Ectopic expression of RCP induced FAK phosphorylation, which links $\beta 1$ integrin with EGFR and participates in a positive regulation loop with EGFR. Interestingly, we observed for the first time that curcumin attenuates RCP-induced ovarian cancer cell invasion by blocking stabilization of $\beta 1$ integrin and consequently inhibiting FAK and EGFR activation, providing potential biomarkers for ovarian cancer and therapeutic approaches for this deadly disease.
\end{abstract}

\section{Introduction}

Ovarian cancer is highly metastatic disease and the fifth leading cause of cancer-related death among women ${ }^{1}$. Early detection and diagnosis of ovarian cancer might significantly improve the survival rate, but the 5-year survival rate is less than $30-50 \%{ }^{2}$. This deadly disease spreads to various sites, such as the liver, pleura, and lung, and the survival rate of patients is based on their metastatic status ${ }^{3}$.

Metastasis is a multi-step event that includes epithelialto-mesenchymal transition (EMT) ${ }^{4}$. During EMT, the cells lose their epithelial characteristics and acquire a spindle-shaped morphology, initiating invasion and metastasis ${ }^{5}$. Metastatic tumor cells detach from adjacent cells by expressing reduced amounts of E-cadherin. In addition, these mesenchymal cells show higher expression

Correspondence: Do Yeun. Cho (dycho@kyuh.ac.kr) or Hoi Young. Lee (hoi@konyang.ac.kr)

${ }^{1}$ Department of Pharmacology, College of Medicine, Konyang University, Daejeon, Korea

${ }^{2}$ Department of Hematology and Oncology, College of Medicine, Konyang

University, Daejeon, Korea

These authors contributed equally: So Ra Choe, Yu Na Kim. of mesenchymal markers, including Snail, Slug, Zeb1, and Twist1, than epithelial cells ${ }^{6}$.

Rab coupling protein (RCP), known as Rab11 familyinteracting protein 1 (Rab11FIP1), is located within the 8p11-12 chromosomal region that is frequently amplified in breast cancers ${ }^{7}$. Accumulating studies have shown that RCP augments cancer tumorigenesis, invasion, and metastasis $^{7-10}$. Mechanistically, RCP associates with $\beta 1$ integrin and links this integrin with receptor tyrosine kinases, such as EGFR, at recycling endosomes that magnify signaling to activate Ras and Erk ${ }^{11}$. In addition, we recently showed that RCP aggravates cancer cell invasion and metastasis by stabilizing $\beta 1$ integrin and consequently upregulating Slug expression and $\mathrm{EMT}^{10}$.

Growing evidence has shown the potential of natural products to act as cancer therapeutic agents. A naturally occurring component of turmeric, curcumin has been shown to inhibit multiple signaling pathways associated with cancer invasion and metastasis. Notably, curcumin inhibits activation of $\mathrm{FAK}^{12}, \mathrm{NF}-\mathrm{KB}^{13}$, and STAT-3 (refs. ${ }^{14,15}$ ). Curcumin also attenuates 
lysophosphatidic acid ${ }^{15}$ - and epidermal growth factor $(\mathrm{EGF})^{16}$-induced ovarian cancer cell migration.

Focal adhesion kinase (FAK) is a key signaling factor that regulates cancer cell motility. Upon activation by numerous stimuli, including integrin clustering, FAK is associated with various small GTPase proteins (Rho, Rac, Cdc42, and Ras) and Src, leading to alteration in the polymerization or stabilization of actin and microtubule filaments ${ }^{17}$. Additionally, FAK has been shown to aggravate ovarian and breast cancer progression by regulating phosphatidylinositol 3-kinase and activation of AKT signaling ${ }^{18,19}$.

Recent studies have demonstrated that integrin endocytosis regulates FAK signaling and that endosomal FAK signaling increases cancer metastasis ${ }^{20}$. Although it is well documented that RCP-induced $\beta 1$ integrin signaling is closely associated with ovarian cancer cell progression, the detailed underlying mechanism by which RCP induces ovarian cancer cell invasion remains unclear. In the present study, we showed that FAK is implicated in RCPinduced EGFR phosphorylation, leading to ovarian cancer cell invasion. In addition, we demonstrated that FAK links $\beta 1$ integrin with EGFR and participates in a positive regulation loop with EGFR. Finally, and more importantly, we showed for the first time that curcumin efficiently inhibits RCP-induced ovarian cancer invasion by blocking RCP-induced stabilization of $\beta 1$ integrin and consequently inhibiting FAK and EGFR activation.

\section{Materials and methods Reagents}

PF573228, curcumin, cycloheximide (CHX), and G418 were obtained from Sigma-Aldrich (St Louis, MO). Gefitinib was from Selleckchem (Houston, TX). Doxorubicin (DOX) was obtained from Cayman (Ann Arbor, MI). All reagents were of the purest grade available.

\section{Cell culture}

Ovarian cancer SKOV-3, OVCAR-3, and PA-1 cells were purchased from American Type Culture Collection (Manassas, VA) and used between the 10th passage and 30th passage. SKOV-3 and OVCAR-3 cells were maintained in RPMI 1640 medium supplemented with $10 \%$ fetal bovine serum (HyClone, Logan, UT) and 1\% penicillin/streptomycin (HyClone). PA-1 cells were maintained in MEM supplemented with $10 \%$ fetal bovine serum. All cells were incubated at $37^{\circ} \mathrm{C}$ under $5 \% \mathrm{CO}_{2}$ in a humidified incubator.

\section{siRNA and plasmid DNA transfection}

SKOV-3, OVCAR-3, and PA-1 cells were transiently transfected with Lipofectamine 3000 or RNAiMAX according to the manufacturer's instructions (Invitrogen, Carlsbad, CA). SKOV-3 cells were stably transfected with $\mathrm{RCP}(4 \mu \mathrm{l})$ by utilizing Lipofectamine $3000(10 \mu \mathrm{l})$ in a six-well plate and selecting for stable transfectants with G418 $(400 \mu \mathrm{l} / \mathrm{ml})$. The empty pEGFP-C3 vector was used as a negative control. siRNAs against FAK (PTK2), $\beta 1$ Integrin (No. 1 and No. 2), ILK (No. 1 and No. 2), Rab11, and Rab25 were purchased from SigmaAldrich. Control scrambled siRNA was from Invitrogen.

\section{Immunoblotting}

Proteins were extracted using RIPA lysis buffer (0.5 M Tris, Triton X-100, Na-deoxycholate, 10\% SDS, $\mathrm{NaCl}$ and $0.5 \mathrm{M}$ EDTA) containing a complete protease inhibitor cocktail tablet (Roche, Indianapolis, IN). Total cell lysates were measured using the Pierce BCA Protein Assay Kit (Thermo Fisher Scientific Inc., Rockford, IL) and were resolved by $8 \%$ sodium dodecyl sulfate polyacrylamide gel electrophoresis and transferred to polyvinylidene difluoride membranes (Millipore, Temecula, CA). The membranes were blocked with 5\% nonfat dried milk in TBST (Tris-buffered saline with $0.1 \%$ Tween-20), incubated for $2 \mathrm{~h}$, and then probed with the indicated primary antibodies overnight at $4{ }^{\circ} \mathrm{C}$. Next, the membranes were washed twice with washing buffer and incubated with secondary antibodies (rabbit, mouse; Thermo Fisher Scientific) for $2 \mathrm{~h}$ at room temperature. Then, the immunoreactive bands were visualized by enhanced chemiluminescence (Thermo Fisher Scientific) using ImageQuant 400 (GE Healthcare, Buckinghamshire, UK). Antibody for E-cadherin (610182) was obtained from BD Bioscience (San Jose, CA). Anti-FAK (557), EGFR (03), $\beta 1$ Integrin $(53,711)$, and GAPDH $(25,778)$ antibodies were purchased from Santa Cruz Biotechnology (Santa Cruz, Texas, CA). Anti-RCP (12,849), p-FAK (3283 S), Slug (9585), and p-EGFR (4407) antibodies were from Cell Signaling Technology (Danvers, MA). Anti-ILK $(76,468)$ was purchased from Abcam (Cambridge, MA).

\section{Immunofluorescence}

The cells were fixed with cold methanol prior to being blocked in $1 \%$ phosphate-buffered saline (PBS). Immunofluorescence was carried out with anti-p-FAK $(16,665$, 1:100; Santa Cruz Biotechnology), E-cadherin (7870, 1:100; Santa Cruz Biotechnology), Slug (15,391, 1:100; Santa Cruz Biotechnology), and $\beta 1$ integrin (53,771, 1:100; Santa Cruz Biotechnology) antibodies overnight. The cells were washed with ice-cold PBS three times and incubated with Cy2-conjugated donkey anti-goat IgG (305-155-003, 1:500; Jackson ImmunoResearch, West Grove, PA), Cy2conjugated goat anti-rabbit IgG (111-225-144, 1:500; Jackson ImmunoResearch), Cy3-conjugated goat antirabbit IgG (111-165-003, 1:500; Jackson ImmunoResearch), and Cy2-conjuated goat anti-mouse (115-225003, 1:500; Jackson ImmunoResearch) at room temperature. The cells were examined by confocal microscopy ( $\times 200$ and $\times 400$, LSM710; Carl Zeiss, Jena, Germany). 


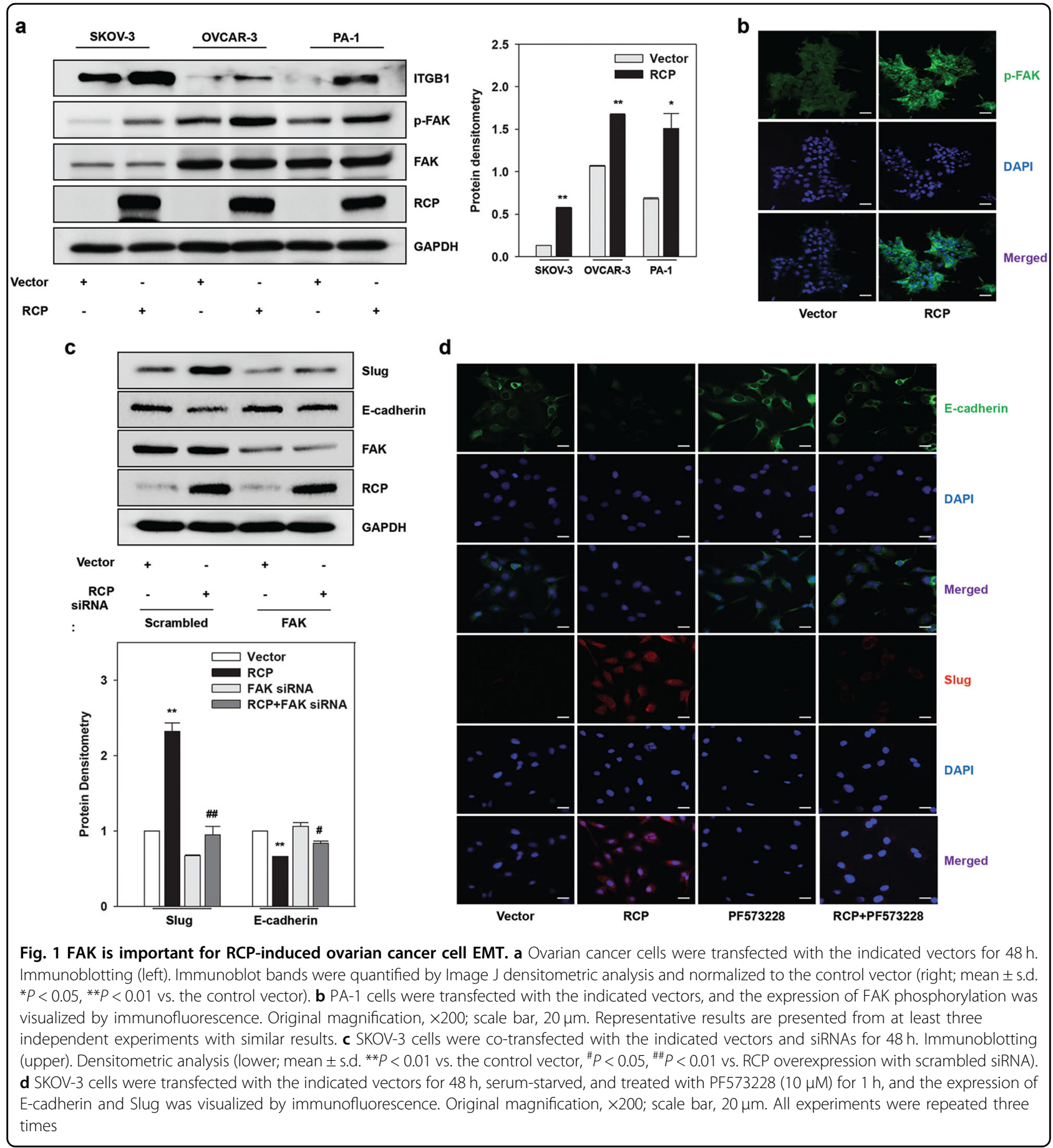

\section{In vitro invasion assay}

In vitro invasion assays were performed in triplicate with an invasion assay kit with Matrigel-coated inserts (BD Bioscience, San Jose, CA), as described previously ${ }^{21}$. A total of $1 \times 10^{6}$ SKOV-3 and PA- 1 cells per well were added to the upper compartment of the invasion chamber with or without pharmacologic inhibitors. To the lower compartment, we added serum-free conditioned medium. After incubation for $18 \mathrm{~h}$ at $37^{\circ} \mathrm{C}$, the invaded cells were sequentially fixed, stained with Diff-Quik reagents (Dade Behring Inc., Newark, DE), and quantitated by counting the number of cells in five random highpower fields for each replicate $(\times 200)$ with a light microscope.

\section{Three-dimensional Matrigel culture}

We established three-dimensional (3-D) cultures with Matrigel (BD Biosciences). The SKOV-3 cells were 
suspended in 2\% (v/v) Matrigel and seeded over a layer of polymerized $100 \%$ Matrigel at $3 \times 10^{4}$ cells $/ \mathrm{ml}$ in eightwell chamber slides (Nunc, Littleton, CO). Cell culture medium was changed every second day. Cultures were analyzed after 7 days of cultivation.

\section{Cell viability assay}

Serum-starved cells were treated with or without curcumin and DOX for $24 \mathrm{~h}$ and then washed with PBS, followed by addition of $5 \mathrm{mg} / \mathrm{ml}$ MTT [(3,4, 5-dimetylathiazol-2yl)-5-diphenyl-tetrazolium bromide].
After incubation for $2 \mathrm{~h}$, cells were washed with PBS and combined with $100 \mu \mathrm{l}$ dimethyl sulfoxide. The absorbance was measured at $540 \mathrm{~nm}$ using an ELISA plate reader (Bio-Rad Laboratories, Hercules, CA). The results are expressed as the percent decrease in cell viability compared with the control.

\section{Statistical analysis}

Data are shown as the mean \pm standard deviation (s.d.). Differences between two groups were assessed using Student's $t$-test. Differences among three or more groups

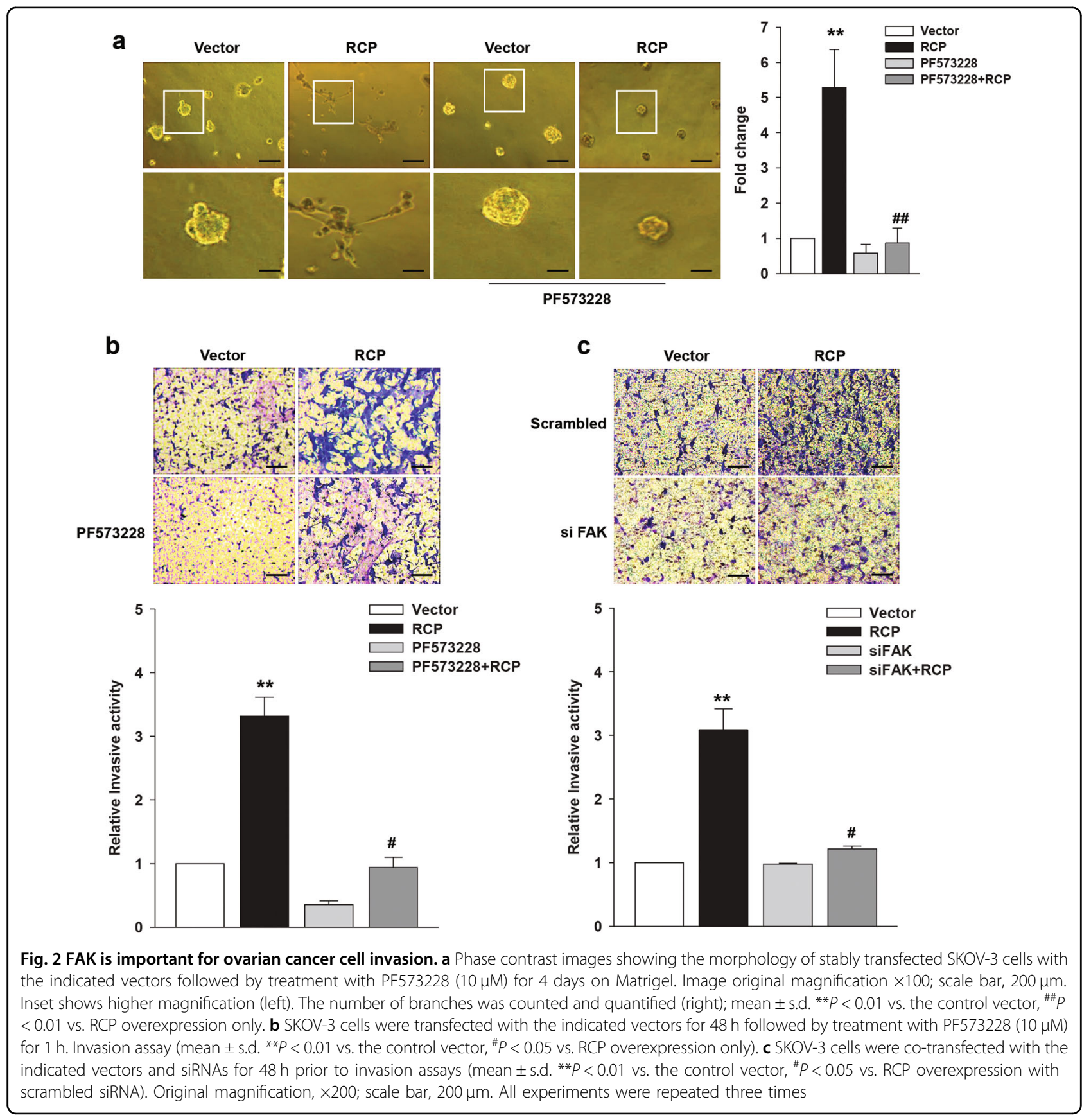


were evaluated by analysis of variance followed by Bonferroni multiple comparison tests.

\section{Results}

FAK is important for RCP-induced ovarian cancer cell EMT

Previously, we showed that RCP induces ovarian cancer cell invasion through stabilizing the $\beta 1$ integrin protein and inducing Slug expression ${ }^{10}$. To identify the role of FAK in RCP-induced ovarian cancer invasion, we first determined whether RCP activates FAK in ovarian cancer cells. Indeed, ectopic expression of RCP strongly increased FAK phosphorylation at Tyr397 to create a binding motif for various $\mathrm{SH} 2$-domain-containing proteins, including Src (Fig. 1a). Immunofluorescence data showed that RCP induces FAK phosphorylation (Fig. 1b). We next addressed the role of FAK in EMT of
RCP-induced ovarian cancer cells. As reported previously ${ }^{10}$, RCP induced Slug expression (Fig. 1c). However, silencing of FAK expression abolished RCPinduced Slug expression along with recovery of Ecadherin expression that was reduced by RCP (Fig. 1c). Furthermore, treatment of the cells with a pharmacological inhibitor of FAK, PF573228, showed similar results to inhibition of RCP-induced Slug expression (Supplementary Figure 1). Immunofluorescence analysis also demonstrated that inhibition of FAK activation by PF573228 abolishes RCP-induced Slug expression and subsequently increases E-cadherin expression (Fig. 1d). Therefore, these data indicate that FAK is implicated in RCP-induced ovarian cancer cell EMT.

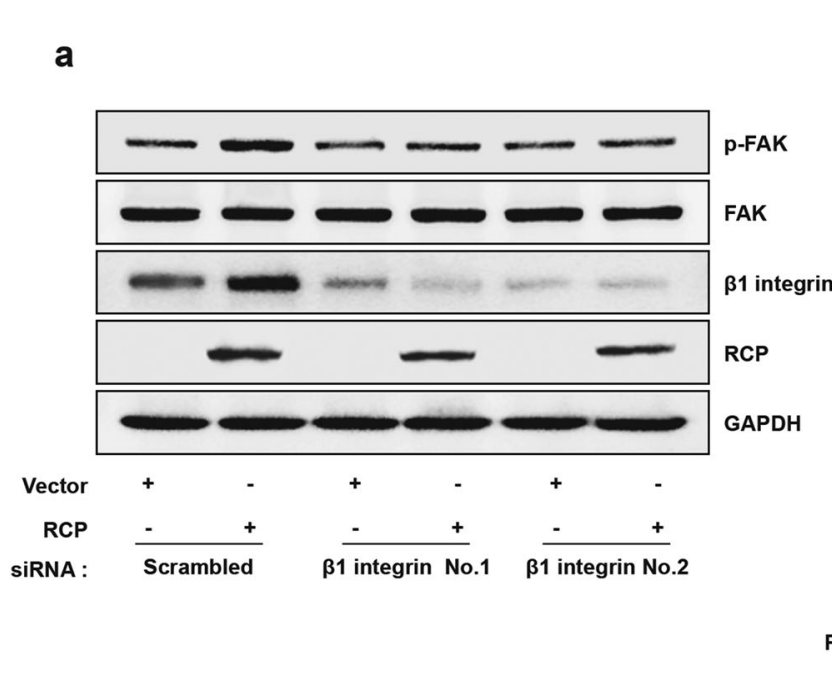

C

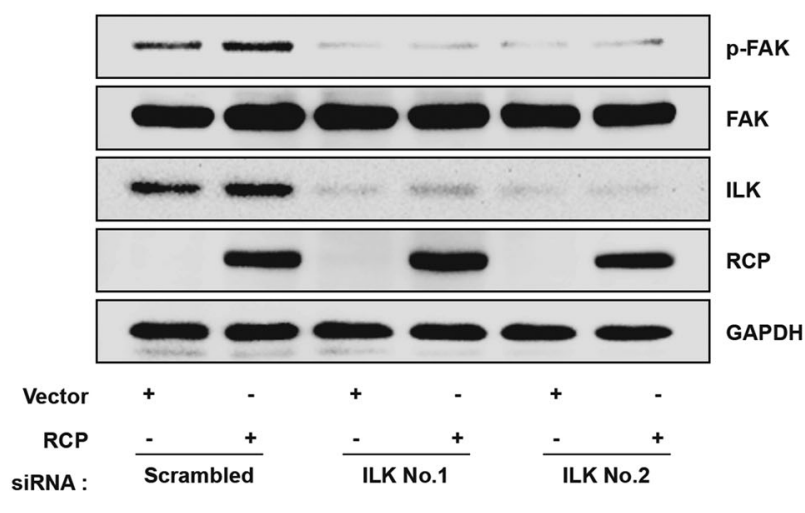

b

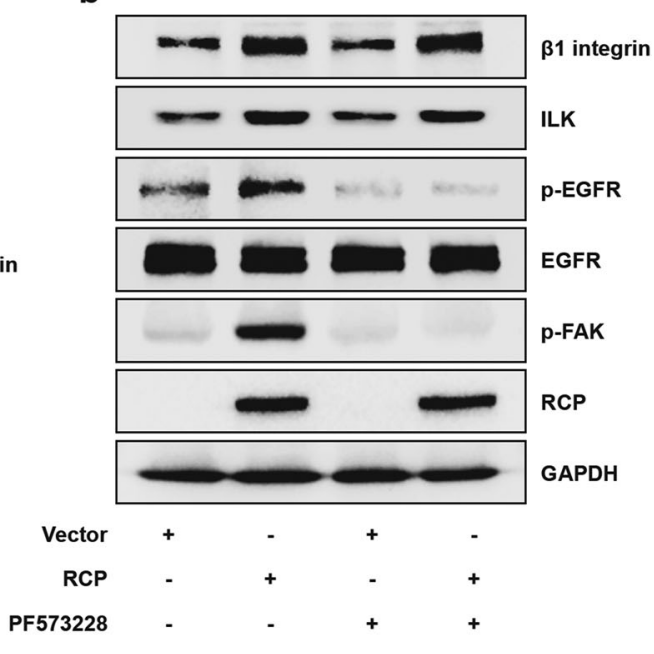

d

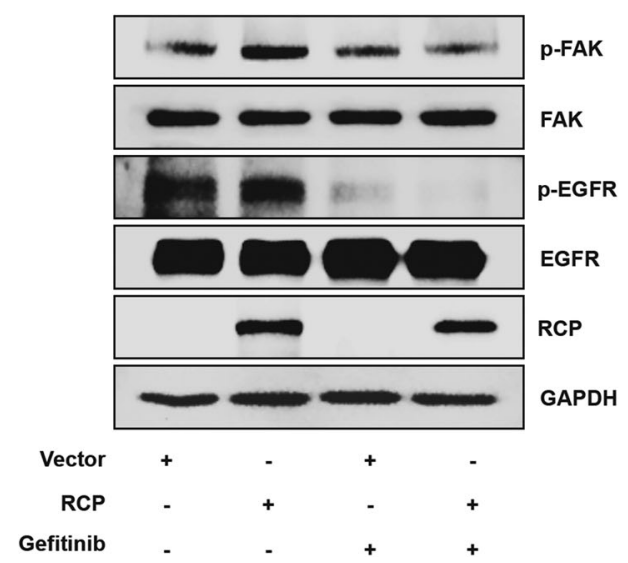

Fig. 3 FAK links $\beta 1$ integrin with EGFR. a SKOV-3 cells were co-transfected with the indicated vectors and siRNAs for $48 \mathrm{~h}$. $\mathbf{b}$ SKOV-3 cells were transfected with the indicated vectors for $48 \mathrm{~h}$, serum-starved, and treated with PF573228 (10 $\mu \mathrm{M})$ for $1 \mathrm{~h}$. c SKOV-3 cells were co-transfected with the indicated vectors and siRNAs for $48 \mathrm{~h}$. $\mathbf{d}$ SKOV-3 cells were transfected with the indicated vectors for $48 \mathrm{~h}$, serum-starved, and treated with gefitinib (1 $\mu \mathrm{M})$ for $1 \mathrm{~h}$. Immunoblotting. All experiments were repeated three times 
a

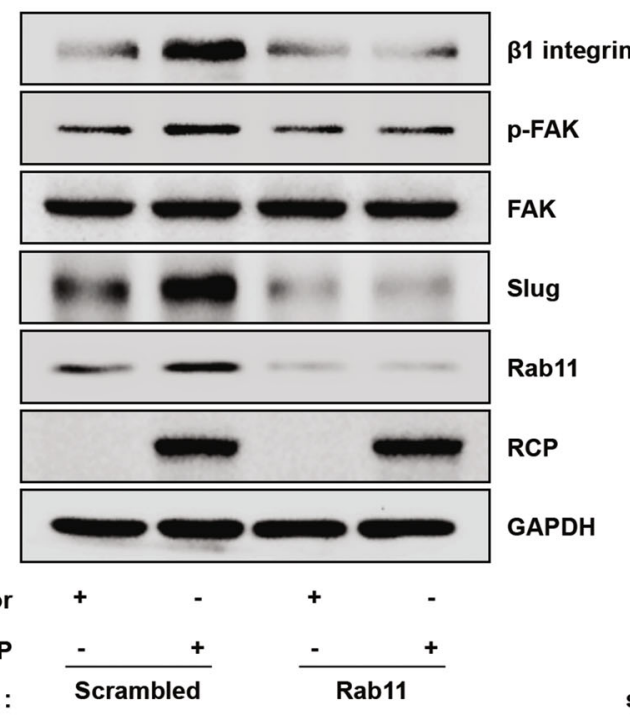

b
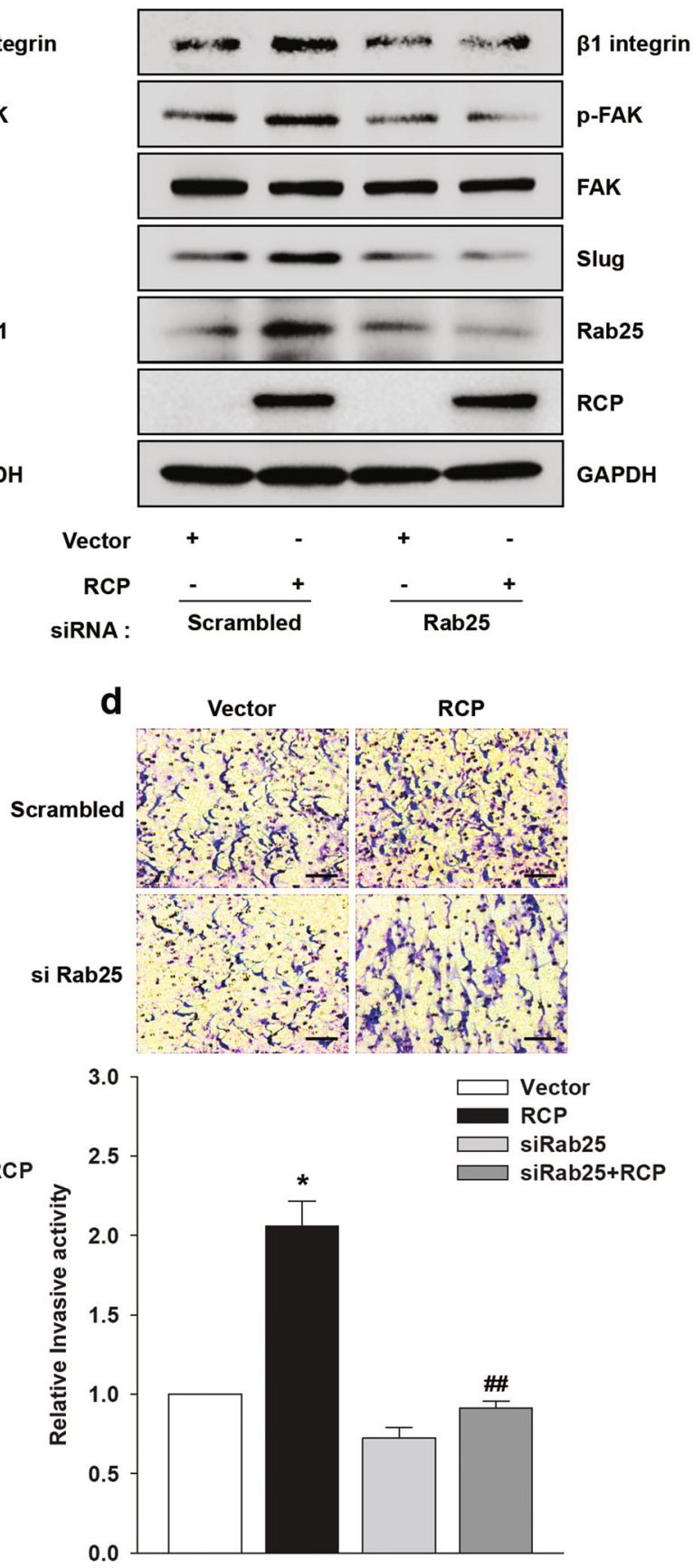

Fig. 4 Rab11 and Rab25 are required for RCP-induced FAK phosphorylation. a, b SKOV-3 cells were co-transfected with the indicated vectors and siRNAs for $48 \mathrm{~h}$. Immunoblotting and $\mathbf{c}$, $\mathbf{d}$ invasion assays (mean \pm s.d. ${ }^{*} P<0.05,{ }^{* *} P<0.01$ vs. the control vector, ${ }^{\#} P<0.05$, ${ }^{\# \#} P<0.01$ vs. RCP overexpression with scrambled siRNA). Original magnification, $\times 200$; scale bar, $200 \mu \mathrm{m}$. All experiments were repeated three times

FAK is important for ovarian cancer cell invasion

Since RCP is known to induce invasive migration of cancer cells ${ }^{9,10}$, we next determined the role of FAK in RCP-induced cancer cell invasion. Cells transfected with RCP substantially increased the number of invasive foci on 3-D Matrigel compared those transfected with the control vector (Fig. 2a). However, pretreatment of the cells with PF573228 abolished the RCP-induced increase in invasive foci, indicating that FAK is implicated in RCPinduced morphological changes of ovarian cancer cells. In addition, pretreatment of the cells with PF573228 (Fig. 2b and Supplementary Figure 2a) or transfection of the cells 


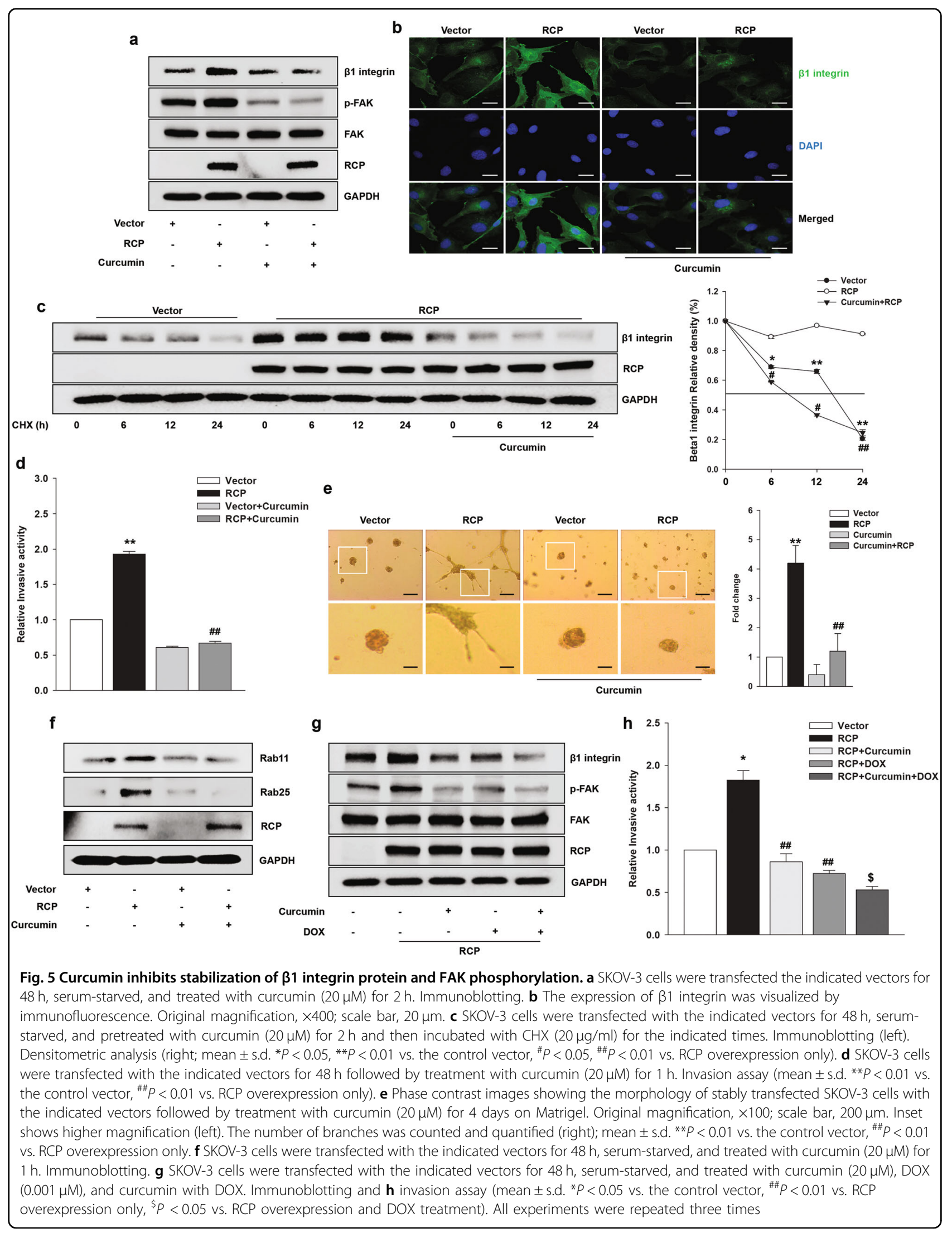


with FAK siRNA (Fig. 2c and Supplementary Figure 2b) significantly inhibited RCP-induced ovarian cancer cell invasion, indicating that FAK plays an indispensable role in RCP-induced ovarian cancer cell invasion.

\section{FAK links $\beta 1$ integrin with EGFR}

Previous studies noted that FAK is located in the recycling endosome and activated by $\beta 1$ integrin $^{22}$. However, the downstream target of FAK required for cancer cell invasion has not been identified. To determine the location of FAK in the RCP-induced signaling cascade, we transfected the cells with two different $\beta 1$ integrin siRNAs. Silencing of $\beta 1$ integrin expression strongly reduced RCP-induced FAK phosphorylation (Fig. 3a). Conversely, treatment of the cells with PF573228 did not affect RCP-induced $\beta 1$ integrin expression (Fig. 3b), indicating that FAK is located downstream of $\beta 1$ integrin. We observed very similar results with silencing of ILK; transfection of the cells with ILK siRNAs substantially inhibited RCP-induced FAK phosphorylation (Fig. 3c). However, treatment of the cells with PF573228 and a pharmacological inhibitor of EGFR, gefitinib, abrogated RCP-induced EGFR, and FAK phosphorylation, respectively (Fig. 3b, d). These findings indicate that FAK links $\beta 1$ integrin with EGFR and participates in the positive regulation loop with EGFR in RCP-induced ovarian cancer cell invasion.

\section{Rab11 and Rab25 are required for RCP-induced FAK phosphorylation}

RCP has been shown to interact with Rab11 or Rab25 (refs. ${ }^{23,24}$ ). In addition, Rab11 and Rab25 augment ovarian cancer cell invasion by recycling both integrins and $\mathrm{EGFR}^{25,26}$. Therefore, we decided to investigate whether Rab11 and Rab25 are prerequisite for RCP-induced FAK phosphorylation. Indeed, silencing of Rab11 or Rab25 expression markedly reduced $\mathrm{RCP}$-induced $\beta 1$ integrin expression and phosphorylation of FAK and EGFR (Fig. 4a, b). In addition, we observed that RCP induces the expression of Rab11 (Fig. 4a) and Rab25 (Fig. 4b). More importantly, Rab11 or Rab25 siRNA completely inhibited RCP-induced ovarian cancer cell invasion (Fig. 4c, d). Therefore, these data indicate that RCP regulates the levels of Rab11 and Rab25 and that both Rab11 and Rab25 are essential for RCP-induced ovarian cancer cell invasion.

\section{Curcumin inhibits stabilization of $\beta 1$ integrin and FAK phosphorylation}

Since curcumin has been shown to inhibit FAK phosphorylation in various cancer cells ${ }^{12,15,27}$, we next investigated its effect on RCP-induced FAK phosphorylation. Treatment of the cells with $20 \mu \mathrm{M}$ curcumin (Supplementary Figure 3) effectively inhibited RCP-induced FAK phosphorylation (Fig. 5a). Interestingly, we observed that curcumin also reduces RCP-induced $\beta 1$ integrin expression. Immunofluorescence analysis confirmed the inhibitory effects of curcumin on RCP-induced $\beta 1$ integrin expression (Fig. 5b). Since our previous report demonstrated that RCP stabilizes $\beta 1$ integrin $\operatorname{protein}^{10}$, we next explored whether curcumin affects the stability of $\beta 1$ integrin. When the cells were treated with $\mathrm{CHX}$ to block de novo protein synthesis, RCP increased the half-life of $\beta 1$ integrin protein (Fig. 5c). However, treatment of the cells with curcumin significantly inhibited the RCPinduced increase in the half-life of $\beta 1$ integrin. In addition, curcumin efficiently attenuated RCP-induced cancer cell invasion (Fig. 5d) and the number of invasive foci on 3-D Matrigel (Fig. 5e). These data, therefore, indicate that curcumin inhibits RCP-induced cancer cell invasion by reducing the recycling of $\beta 1$ integrin and subsequent inactivation of FAK and EGFR. To determine the mechanism underlying curcumin-mediated reduction of $\mathrm{RCP}$-induced recycling of $\beta 1$ integrin, we compared the effects of curcumin on the levels of Rab11 and Rab25 in RCP-overexpressing cells. Interestingly, curcumin dramatically downregulated the expression of Rab11 and Rab25 induced by RCP to the level of the control (Fig. 5f), suggesting that losing partners of RCP reduces the endosome recycling of $\beta 1$ integrin to the plasma membrane.

Given that DOX in a PEGylated liposomal nanoencapsulation has been used for recurrent ovarian cancer ${ }^{28}$, we investigated whether curcumin potentiates the inhibitory effect of DOX on RCP-induced ovarian cancer cell invasion. Intriguingly, we observed increased inhibition of RCP-induced $\beta 1$ integrin expression (Fig. 5g) and ovarian cancer cell invasion (Fig. 5h) when the cells were treated with both curcumin and DOX at a dose with an over $85 \%$ survival rate (Supplementary Figure 4) compared with treatment with single agent, suggesting that DOX potentiates the inhibitory effects of curcumin on RCPinduced $\beta 1$ integrin expression and FAK phosphorylation as well as the consequent ovarian cancer cell invasion.

\section{Discussion}

Herein, we elucidated the essential role of FAK in RCPinduced cancer cell invasion and its inhibition by curcumin. Ectopic expression of RCP induced FAK phosphorylation, which creates a positive feedback loop with EGFR. In addition, FAK acts as a link between the $\beta 1$ integrin/ ILK axis and EGFR, leading to ovarian cancer cell EMT and invasion. Furthermore, our present data demonstrated that both Rab11 and Rab25 are essential for RCPinduced FAK phosphorylation and that curcumin inhibits RCP-induced FAK phosphorylation and ovarian cancer invasion by blocking stabilization of $\beta 1$ integrin. Finally, we provided evidence that DOX potentiates the inhibitory 
effect of curcumin on RCP-induced stabilization of $\beta 1$ integrin and on FAK phosphorylation, leading to attenuation of RCP-induced ovarian cancer cell invasion.

RCP was initially identified as a Rab11/Rab4/Rab25interacting protein with putative physiological roles in endosomal trafficking and receptor sorting ${ }^{23,29,30}$. RCP was co-localized with Rab11 and required for recycling of integrin a5 $\beta 1$ at the tips of the long pseudopods ${ }^{31}$. Furthermore, RCP was proposed to be a key component of an integrin recycling system through binding with Rab25 and Rab11 (ref. ${ }^{32}$ ). Recently, the Rab25 and RCP expression levels were shown to be coordinately regulated with a positive feedback loop ${ }^{33}$. Consistent with this finding, we demonstrated in the present study that RCP induces the expression of Rab11 and Rab25, suggesting that it amplifies the common signaling of RCP, Rab11 and Rab25 for ovarian cancer cell invasion.

We recently showed that RCP induces ovarian cancer cell EMT through coordinate activation of the $\beta 1$ integrin/ILK/EGFR signaling axis and Slug expression ${ }^{10}$. Ectopic expression and silencing of RCP induced and reduced ovarian cancer cell EMT, respectively ${ }^{10}$. In addition, FAK is located downstream of $\beta 1$ integrin $^{34}$ and implicated in ovarian cancer cell metastasis ${ }^{35}$. Our present study further elucidated the underlying mechanism of RCP-induced ovarian cancer cell invasion and identified the downstream target of FAK. We show that FAK positioned between ILK and EGFR activates EGFR, which in turn phosphorylates FAK, leading to a positive feedback loop in RCP-induced ovarian cancer cell invasion. We note that these results should be verified in physiological conditions.

Curcumin was shown to have anti-cancer and antimetastatic properties. In addition to reducing expression of various metastasis-related factors ${ }^{36}$, curcumin was recently shown to inhibit EMT of breast cancer stem cells by suppressing Slug expression ${ }^{37}$. Furthermore, curcumin was reported to suppress IL- $1 \beta$-induced $\beta 1$ integrin expression in human chondrocytes ${ }^{38}$. Unexpectedly, our present data showed that curcumin hampers RCPinduced recycling of $\beta 1$ integrin, thereby blocking activation of FAK and the EGFR/Slug signaling axis and ovarian cancer cell invasion. The underlying mechanism of reduced recycling of RCP-induced $\beta 1$ integrin by curcumin appears to be caused by downregulation of a critical partner of RCP, Rab11 and Rab25 expression, since curcumin strongly reduced the levels of RCP-induced Rab11 and Rab25 expression. Moreover, we provide evidence that a combination of DOX with curcumin enhances the anti-invasive properties by reducing the recycling of $\beta 1$ integrin and FAK activation in ovarian cancer cells compared with single treatment, providing a potential therapeutic armament for this deadly disease.
Collectively, our results demonstrated that RCP increases ovarian cancer cell invasion through the $\beta 1$ integrin/ILK/FAK and EGFR signaling pathway and Slug expression and that curcumin efficiently reduces RCPinduced stabilization of $\beta 1$ integrin protein, leading to attenuation of RCP-induced ovarian cancer cell invasion.

\section{Acknowledgements}

This study was supported by a grant from the Basic Science Research Program through the National Research Foundation of Korea (NRF) funded by the Ministry of Education, Science and Technology [NRF-2016R1D1A1B04931788, NRF-2017R1E1A1A01074091].

\section{Conflict of interest}

The authors declare that they have no conflict of interest.

\section{Publisher's note}

Springer Nature remains neutral with regard to jurisdictional claims inpublished maps and institutional affiliations.

Supplementary information accompanies this paper at https://doi.org/ 10.1038/s12276-018-0078-1.

Received: 17 October 2017 Revised: 26 January 2018 Accepted: 13 February 2018.

Published online: 27 April 2018

\section{References}

1. Ozols, R. F. et al. Focus on epithelial ovarian cancer. Cancer Cell 5, 19-24 (2004).

2. Zhou, E. S., Partridge, A. H., Syrjala, K. L., Michaud, A. L. \& Recklitis, C. J. Evaluation and treatment of insomnia in adult cancer survivorship programs. J. Cancer Surviv. 11, 74-79 (2017).

3. Cormio, G. et al. Distant metastases in ovarian carcinoma. Int. Gynecol. Cancer 13, 125-129 (2003).

4. Larue, L. \& Bellacosa, A. Epithelial-mesenchymal transition in development and cancer: role of phosphatidylinositol 3' kinase/AKT pathways. Oncogene 24, 7443-7454 (2005).

5. Vergara, D. et al. Epithelial-mesenchymal transition in ovarian cancer. Cancer Lett. 291, 59-66 (2010).

6. Tomaskovic-Crook, E., Thompson, E. W. \& Thiery, J. P. Epithelial to mesenchymal transition and breast cancer. Breast Cancer Res. 11, 213 (2009).

7. Zhang, J. et al. RCP is a human breast cancer-promoting gene with Rasactivating function. J. Clin. Invest. 119, 2171-2183 (2009).

8. Morello, $\mathrm{V}$. et al. beta1 integrin controls EGFR signaling and tumorigenic properties of lung cancer cells. Oncogene 30, 4087-4096 (2011).

9. Jacquemet, G. et al. RCP-driven alpha5beta1 recycling suppresses Rac and promotes RhoA activity via the RacGAP1-IQGAP1 complex. J. Cell Biol. 202, 917-935 (2013).

10. Hwang, M. H. et al. RCP induces Slug expression and cancer cell invasion by stabilizing beta1 integrin. Oncogene 36, 1102-1111 (2017).

11. Mills, G. B., Jurisica, I., Yarden, Y. \& Norman, J. C. Genomic amplicons target vesicle recycling in breast cancer. J. Clin. Invest. 119, 2123-2127 (2009).

12. Leu, T. H., Su, S. L., Chuang, Y. C. \& Maa, M. C. Direct inhibitory effect of curcumin on Src and focal adhesion kinase activity. Biochem. Pharmacol. 66, 2323-2331 (2003)

13. Aggarwal, S. et al. Curcumin (diferuloylmethane) down-regulates expression of cell proliferation and antiapoptotic and metastatic gene products through suppression of IkappaBalpha kinase and Akt activation. Mol. Pharmacol. 69, 195-206 (2006).

14. Bharti, A. C., Donato, N. \& Aggarwal, B. B. Curcumin (diferuloylmethane) inhibits constitutive and IL-6-inducible STAT3 phosphorylation in human multiple myeloma cells. J. Immunol. 171, 3863-3871 (2003).

15. Seo, J. H. et al. Lysophosphatidic acid induces STAT3 phosphorylation and ovarian cancer cell motility: their inhibition by curcumin. Cancer Lett. 288, 50-56 (2010). 
16. Ji, C. et al. Curcumin attenuates EGF-induced AQP3 up-regulation and cell migration in human ovarian cancer cells. Cancer Chemother. Pharmacol. 62 , 857-865 (2008).

17. Mitra, S. K., Hanson, D. A. \& Schlaepfer, D. D. Focal adhesion kinase: in command and control of cell motility. Nat. Rev. Mol. Cell Biol. 6, 56-68 (2005).

18. Reif, S. et al. The role of focal adhesion kinase-phosphatidylinositol 3-kinase-akt signaling in hepatic stellate cell proliferation and type I collagen expression. J. Biol. Chem. 278, 8083-8090 (2003).

19. You, D. et al. FAK mediates a compensatory survival signal parallel to PI3K-AKT in PTEN-null T-ALL cells. Cell Rep. 10, 2055-2068 (2015).

20. Alanko, J. \& Ivaska, J. Endosomes: emerging platforms for integrin-mediated FAK signalling. Trends Cell Biol. 26, 391-398 (2016).

21. Jeong, K. J. et al. The Rho/ROCK pathway for lysophosphatidic acid-induced proteolytic enzyme expression and ovarian cancer cell invasion. Oncogene 31, 4279-4289 (2012).

22. Nader, G. P., Ezratty, E. J. \& Gundersen, G. G. FAK, talin and PIPKlgamma regulate endocytosed integrin activation to polarize focal adhesion assembly. Nat. Cell Biol. 18, 491-503 (2016).

23. Hales, C. M. et al. Identification and characterization of a family of Rab11interacting proteins. J. Biol. Chem. 276, 39067-39075 (2001)

24. Lindsay, A. J. et al. Rab coupling protein (RCP), a novel Rab4 and Rab11 effector protein. J. Biol. Chem. 277, 12190-12199 (2002).

25. Cheng, K. W. et al. The RAB25 small GTPase determines aggressiveness of ovarian and breast cancers. Nat. Med. 10, 1251-1256 (2004).

26. Tang, B. L. \& Ng, E. L. Rabs and cancer cell motility. Cell Motil. Cytoskeleton 66, 365-370 (2009)

27. Chen, C. C. et al. Curcumin suppresses metastasis via Sp-1, FAK inhibition, and E-Cadherin upregulation in colorectal cancer. Evid. Complement Altern. Med 2013, 541695 (2013).

28. Engelberth, S. A., Hempel, N. \& Bergkvist, M. Development of nanoscale approaches for ovarian cancer therapeutics and diagnostics. Crit. Rev. Oncog. 19, 281-315 (2014).
29. Meyers, J. M. \& Prekeris, R. Formation of mutually exclusive Rab11 complexes with members of the family of Rab11-interacting proteins regulates Rab11 endocytic targeting and function. J. Biol. Chem. 277, 49003-49010 (2002)

30. Peden, A. A. et al. The RCP-Rab11 complex regulates endocytic protein sorting. Mol. Biol. Cell. 15, 3530-3541 (2004).

31. Caswell, P. T. et al. Rab-coupling protein coordinates recycling of alpha5beta1 integrin and EGFR1 to promote cell migration in 3D microenvironments. J. Cell Biol. 183, 143-155 (2008).

32. Agarwal, R., Jurisica, I., Mills, G. B. \& Cheng, K. W. The emerging role of the RAB25 small GTPase in cancer. Traffic 10, 1561-1568 (2009).

33. Mitra, S. et al. Rab25 acts as an oncogene in luminal B breast cance and is causally associated with Snail driven EMT. Oncotarget 7, 40252-40265 (2016).

34. Buczek-Thomas, J. A., Chen, N. \& Hasan, T. Integrin-mediated adhesion and signalling in ovarian cancer cells. Cell Signal. 10, 55-63 (1998).

35. Chen, C. H. et al. MUC20 promotes aggressive phenotypes of epithelial ovarian cancer cells via activation of the integrin beta1 pathway. Gynecol. Oncol. 140, 131-137 (2016).

36. Kunnumakkara, A. B., Anand, P. \& Aggarwal, B. B. Curcumin inhibits proliferation, invasion, angiogenesis and metastasis of different cancers through interaction with multiple cell signaling proteins. Cancer Lett. 269, 199-225 (2008).

37. Mukherjee, S. et al. Curcumin inhibits breast cancer stem cell migration by amplifying the E-cadherin/beta-catenin negative feedback loop. Stem Cell Res. Ther. 5, 116 (2014).

38. Shakibaei, M., Schulze-Tanzil, G., John, T. \& Mobasheri, A. Curcumin protects human chondrocytes from IL-11 beta-induced inhibition of collagen type II and beta1-integrin expression and activation of caspase-3: an immunomorphological study. Ann. Anat. 187, 487-497 (2005). 